\title{
Optical Resolution Photoacoustic Imaging of Multiple Probes via Single Fiber Laser with Independently Adjustable Parameters
}

\author{
Seydi Yavas ${ }^{1}$, E.A. Kipergil ${ }^{1}$, N. Uluc ${ }^{1}$, A Demirkiran ${ }^{1}$, T. Kayikcioglu ${ }^{1}$, H.S. Salman ${ }^{2}$, S.G. Karamuk ${ }^{3,4}$, \\ M. B. Unlu ${ }^{1}$, F.O. Ilday ${ }^{3}$ \\ 1. Department of Physics, Bogazici University, 34342, Istanbul, Turkey \\ 2. FiberLAST Inc., 06800, Ankara, Turkey \\ 3. Department of Electrical Engineering, Bilkent University, 06800, Ankara, Turkey. \\ 4. Lumos Laser Ltd., 06500, Ankara, Turkey
}

Photoacoustic microscopy (PAM) is a promising imaging modality that combines optical and ultrasound imaging. It combines the advantages of high ultrasonic spatial resolution and high optical contrast. When a short laser pulse illuminates the tissue, absorbed light leads to an acoustic emission via thermoelastic expansion [1]. The laser system needs to generate short enough pulses, i.e., several nanoseconds, to create photoacoustic signals with high efficiency and emit wavelengths in the visible range to excite tissue chromophores in their absorption peaks. To increase penetration depth of imaging, it is also desirable to utilize a wavelength in the NIR range, from 600 to $1200 \mathrm{~nm}$, where biological tissues are relatively transparent.

Here, we developed a tunable fiber based laser system producing nanosecond pulses, covering the spectrum from $450 \mathrm{~nm}$ to $1100 \mathrm{~nm}$, specifically for PAM and tested it for imaging with multiple photoacoustic probes inside microfluidic channels. The laser system can be examined in three subsystems; i) fiber laser ii) supercontinuum iii) harmonic generator. The supercontinuum part of the laser is all fiber-integrated; guided-beam-propagation renders it misalignment-free and mostly immune to mechanical perturbations. Total supercontinuum output power is over $1 \mathrm{~W}$, and visible output power is around $270 \mathrm{~mW}$ at $65 \mathrm{kHz}$ repetition rate corresponding to $4 \mu \mathrm{J}$ pulse energy. Free space harmonic generation creates higher pulse energy for a particular band, i.e. $532 \mathrm{~nm}$, and also generates ultraviolet (UV) light with wavelengths of 355 and $266 \mathrm{~nm}$. One of the novelties here is the improvement of wavelength tunability, output power, and pulse energy when fiber-based lasers are benchmarked. The tunability of the laser parameters allows using only one laser for many different PAM applications, and also high repetition rate enables fast scanning. The coverage of near-UV spectrum gives an opportunity to image cell nuclei. As certain morphological changes such as size and shapes irregularities in the nuclei are known indicators of various cancers[2], we believe our system may also be useful for cell nuclei studies as well.

a)

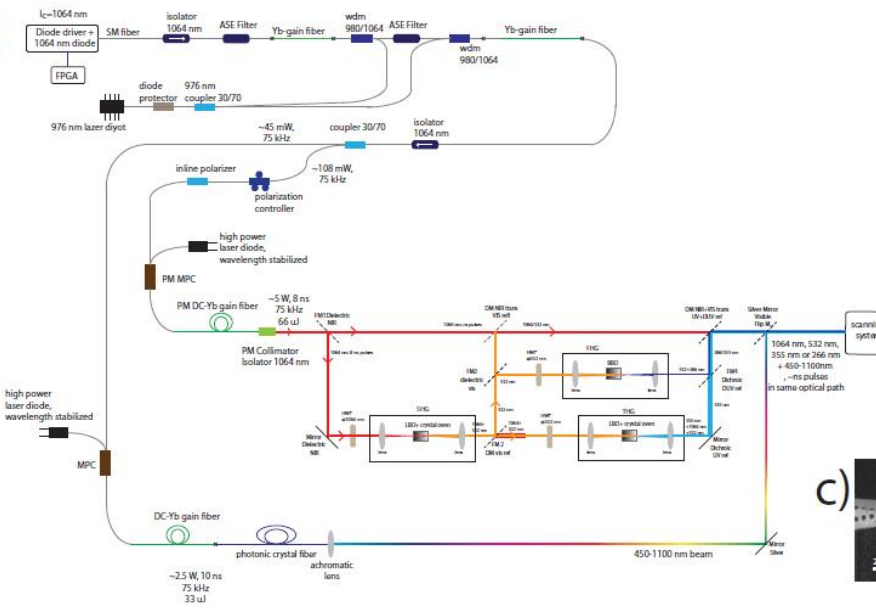

b)

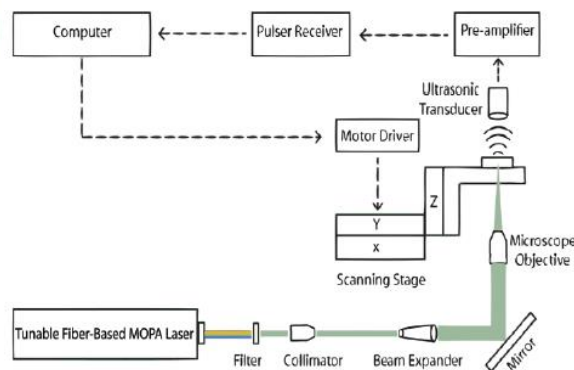

Fig. 1 a) The fiber laser system, supercontinuum and harmonic generation outputs, b) OR-PAM system based on the fiber laser system c) PAM image of Bombay Indian Ink inside microfluidic channels, excitation wavelength is $680 \mathrm{~nm}$, d) Red blood cells inside microfluidic channels, excitation wavelength is $532 \mathrm{~nm}$

We demonstrated the capability of the laser system in PAM by utilizing it for experiments for different probes inside home developed microfluidic channels. In Figure 1c, microfluidic chip filled with Indian Ink that is excited by the $680 \mathrm{~nm}$ light filtered from supercontinuum part of the laser can be seen. In Figure 1d, red blood cells that are excited by the $532 \mathrm{~nm}$ harmonic generation output of the same system is imaged.

\section{References}

[1] Xu, M., and L.V. Wang. "Photoacoustic imaging in biomedicine." Rev. Sci. Instrum. 77.4, 041101 (2006) 041101.

[2] Yao, D.-K., Chen, R., Maslov, K., Zhou, Q. \& Wang, L. V. "Optimal ultraviolet wavelength for in vivo photoacoustic imaging of cell Nuclei”. J. Biomed. Opt.17, 0560041 (2012) 